\title{
MOLECULAR DISTRIBUTIONS IN GALACTIC SOURCES
}

\author{
HÉLENE R. DICKEL \\ University of Illinois Observatory, Ill., U.S.A.
}

\begin{abstract}
The $4830 \mathrm{MHz}$ absorption line of formaldehyde has been observed in the SNR complex W44 and the $\mathrm{H}$ II region complex W3. The results are presented as maps of apparent optical depth. The formaldehyde is concentrated toward the eastern edge of the shell and reaches a maximum apparent optical depth of 1.5 compared with 0.2 for $\mathrm{OH}$; furthermore the centers of the $\mathrm{H}_{2} \mathrm{CO}$ and $\mathrm{OH}$ concentrations are displaced by about $6^{\prime}$. The velocities of the $\mathrm{H}_{2} \mathrm{CO}$ and $\mathrm{OH}$ agree and both molecular lines have the same velocity gradient across the source.

The $\mathrm{H}_{2} \mathrm{CO}$ absorption profiles for W3 (IC 1795) exhibit at least two narrow components whose average velocity becomes more negative toward the NE. The greatest optical depth occurs where the optical obscurration is high but not at the position of maximum $A_{v}$. A general agreement between the velocities of other molecules and the $\mathrm{H}_{2} \mathrm{CO}$ values is indicated.
\end{abstract}

In order to make models of molecular regions to explain their observed density and velocity structure and to determine their evolution as well as to gain insight into the molecular formation and destruction processes it is necessary to intercompare the distributions of a variety of different molecules in a given source and also compare them relative to the associated radio continuum, infrared sources and dust lanes. Thus far $\mathrm{I}$ have mapped the $\mathrm{OH}$ and $\mathrm{H}_{2} \mathrm{CO}$ absorption in a number of extended continuum sources. The formaldehyde results are now presented for the SNR complex W 44 and the $\mathrm{H}$ II region complex W 3 (IC 1795), (Figures 1 and 2).

The formaldehyde observations were made with the NRAO* 140-ft radio telescope equipped with a cooled $6 \mathrm{~cm}$ parametric amplifier and the 413-channel autocorrelator. Measurements were made at 40 positions in W 44 and 24 positions in. W 3 with integration times of $\frac{1}{2}$ to $1 \mathrm{~h}$ and with $3^{\prime}$ separation between grid points. Concurrent profiles were obtained with $8 \mathrm{kHz}$ and $16 \mathrm{kHz}$ resolution. The corresponding separation between data points was $0.5 \mathrm{~km} \mathrm{~s}^{-1}$ and $1.0 \mathrm{~km} \mathrm{~s}^{-1}$.

The W 44 profiles were adequately fit by one Gaussian curve and the resulting map of apparent optical depth

$$
\left(\tau_{\mathrm{H}_{2} \mathrm{CO}}=-\ln \left[1-\frac{\left|\Delta T_{L}\right|}{T_{c}}\right]\right)
$$

is shown in Figure 1 (solid lines) superposed on an abbreviated version of a $6 \mathrm{~cm}$ continuum map** (dashed lines). For comparison two of the contours from the apparent optical depth map for the $1667 \mathrm{MHz} \mathrm{OH}$ absorption (Goss et al., 1971) are

\footnotetext{
* Operated by Associated Universities, Inc. under contract with the National Science Foundation. ** The $6 \mathrm{~cm}$ contours are an adaptation of Milne's map (1969) which was convolved to an effective resolution of $6^{\prime}$ and converted to $T_{\mathrm{A}}$ by applying the beam effciency factor of 0.81 for the NRAO 140-ft telescope. The formaldehyde line temperatures are on the same temperature scale.
} 


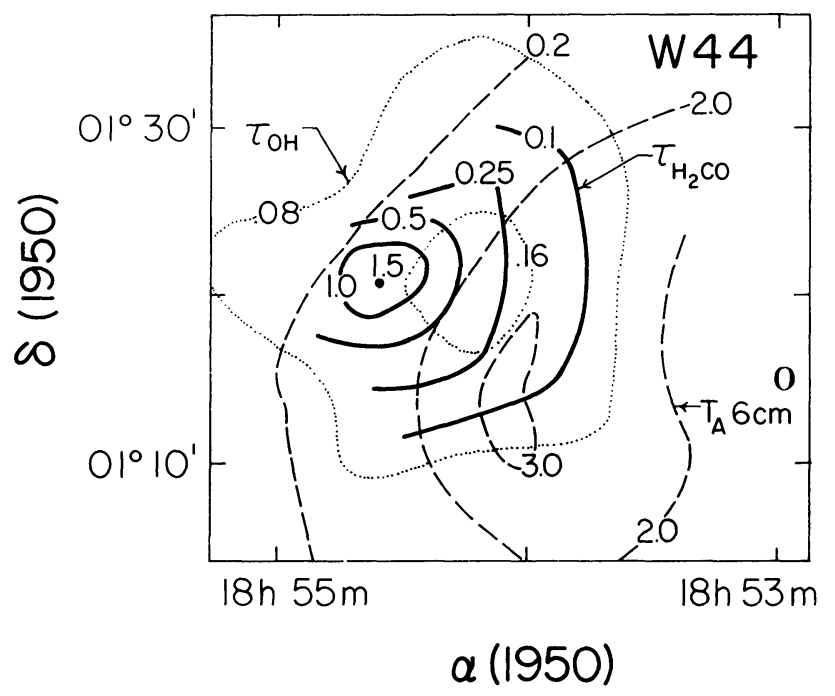

Fig. 1. Map of apparent optical depth for formaldehyde in W 44 (see text).

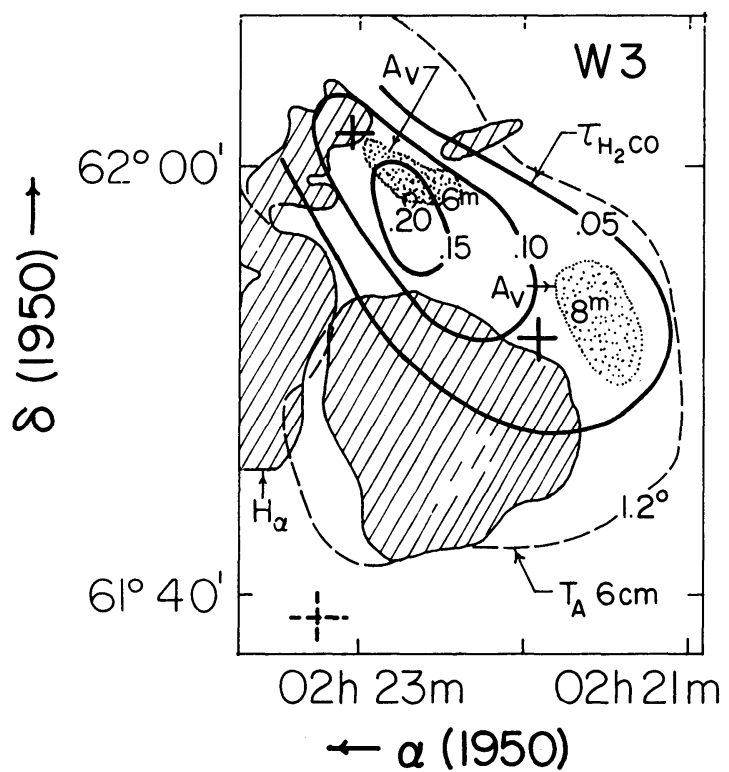

Fig. 2. Map of apparent optical depth for formaldehyde in W 3 (see text).

shown by dotted lines. The formaldehyde is concentrated toward the eastern edge of the shell where the gas compression is highest and no absorption was detected at the secondary continuum peak on the western side (marked by $\mathrm{O}$ on Figure 1). The maximum $\tau_{\mathrm{H}_{2} \mathrm{CO}}$ is 1.5 whereas $\tau_{\mathrm{OH}}$ does not exceed 0.2. The centers of the $\mathrm{H}_{2} \mathrm{CO}$ and $\mathrm{OH}$ concentrations are displaced by about $6^{\prime}$. (These values have not been corrected for resolution effects and may consequently be somewhat altered.) With more limit- 
ed data Whiteoak and Gardner (1972) obtain similar results. The observed $\mathrm{H}_{2} \mathrm{CO}$ and $\mathrm{OH}$ velocities agree surprisingly well, to within the errors of the measurements. There is a velocity gradient across the source with the values becoming more positive toward the edge of the shell as the optical depth increases. Goss et al. (1971) attribute this to a rotation of the cloud of molecules.

Most of the $\mathrm{H}_{2} \mathrm{CO}$ absorption profiles for $\mathrm{W} 3$ show two narrow features with halfintensity widths of about $2.2 \mathrm{~km} \mathrm{~s}^{-1}$ and a separation on the order of $2.0 \mathrm{~km} \mathrm{~s}^{-1}$. There may be an additional weaker feature at the high velocity side. There is a velocity gradient across the source with the more negative velocities toward the NE. The optical depth maps of the individual velocity components are very similar. Thus the solid curves in Figure 2 are only the $\tau_{\mathrm{H}_{2} \mathrm{CO}}$ contours derived from a one-component Gaussian fit to the profiles and ignore the velocity gradient. The dashed line is the $1.2 \mathrm{~K}$ contour of the $6 \mathrm{~cm}$ continuum map of Rubin and Mezger (1970) with the main components $A, B$ and $C$ marked by crosses. The hatched area represents the $\mathrm{H}_{\alpha}$ emission and the two speckled regions indicate where the visual absorption $A_{v}$ exceeds 6 and 8 mag. respectively (Ishida and Kawajiri, 1968). The greatest optical depth for formaldehyde occurs in the optically obscured region but does not coincide with the highest values of $A_{v}$. The velocity gradient from $A$ and $B$ is similar to that found by Rubin and Mezger (1970) for the $\mathrm{H} 109 \alpha$ recombination line but is less complex; the total velocity spread is less and the $\mathrm{H}_{2} \mathrm{CO}$ velocities are generally more positive by about $1.5 \mathrm{~km} \mathrm{~s}^{-1}$. In spite of the different spatial resolutions it is noteworthy that the velocities of the other molecules $\left(\mathrm{H}\right.$ - Chaisson, 1972; $\mathrm{OH}$ - Turner, 1970; $\mathrm{H}_{2} \mathrm{O}-$ Knowles et al., 1969; CO - Penzias et al., 1970; X-ogen - Buhl and Snyder, 1972) near the main $6 \mathrm{~cm}$ continuum peak $(A)$ either agree with the average $\mathrm{H}_{2} \mathrm{CO}$ velocity or with one of the components of the line.

Further analysis and interpretation of these two sources is under way. I am assessing the effects of the finite resolution on the W 44 results. For W 3 I will investigate whether or not the distribution of formaldehyde can be related to the small scale structures of the molecular emission and IR sources (see Wynn-Williams' high resolution maps) (Wynn-Williams et al., 1973). Thus far it appears that a rotating cloud model fits the W 44 data and may also apply to the W3 complex. This is in contrast to the model of an expanding shell of compressed material which we find for the Carina nebula (Dickel and Wall, 1971 and 1972). My planned studies of a number of molecules in many more sources should clarify the variety of conditions existing in these molecular clouds near the different kind of continuum sources and contrast these to the situation in dust clouds which are isolated from any continuum source.

\section{Acknowledgements}

I thank Tom Cram and David Buhl for their generous assistance in processing the data. I appreciated the dedication of George Behrens for keeping the equipment operating.

This research was supported in part by the National Science Foundation. 


\section{References}

Buhl, D. and Snyder, L.: 1972, private communication. Chaisson, E. J.: 1972, Astron. Astrophys. 18, 149.

Dickel, H. R. and Wall, J. V.: 1971, Bull. Am. Astron. Soc. 3, 459.

Dickel, H. R. and Wall, J. V.: 1972, 'Carina Nebula II: $18 \mathrm{~cm} \mathrm{OH}$ Observations and Comparison of the Distribution of $\mathrm{OH}$ and $\mathrm{H}_{2} \mathrm{CO}$ Molecules', in preparation.

Goss, W. M., Caswell, J. L., and Robinson, B. J.: 1971, Astron. Astrophys. 14, 481.

Ishida, K. and Kawajiri, N.: 1968, Publ. Astron. Soc. Japan 20, 95.

Knowles, S. H., Mayer, C. H., Cheung, A. C., Rank, D. M., and Townes, C. H.: 1969, Science 163, 1055.

Milne, D. K.: 1969, Australian J. Phys. 22, 613.

Penzias, A. A., Jefferts, K. B., and Wilson, R. W.: 1970, Astrophys. J. 165, 229.

Rubin, R. H. and Mezger, P. G.: 1970, Astron. Astrophys. 5, 407.

Turner, B. E.: 1970, Astrophys. Letters 6, 99.

Whiteoak, J. B. and Gardner, F. F.: 1972, Astron. Astrophys. 21, 159.

Wynn-Williams, C. G., Becklin, E. E., and Neugebauer, G.: 1973, this volume, p. 459. 